\title{
Structure of a shell made from uranium alloyed with iron and germanium after explosive loading
}

\author{
D.A. Belyaev*, A.S. Aleksandrov, Yu.N. Zuev, E.A. Kozlov, \\ A.E. Shestakov, S.A. Lekomtsev, A.S. Nedosviti, I.L. Svyatov, E.A. Levi \\ Russian Federal Nuclear Center - Zababakhin All-Russia Research Institute of \\ Technical Physics, Snezhinsk, Russia
}

\begin{abstract}
This paper presents results of metallographic examination of a thick-wall spherical shell from uranium alloyed with iron and germanium. This shell is recovered after low-level explosive loading. Light microscopy, hardness measurement, scanning electron microscopy and X-Ray diffraction study were used to investigate the meridional section of the test shell as this section most completely exhibits the whole variety of structural features associated with explosive loading of the material. Processing, presentation, and analysis of experimental data on volumetric distribution of studied physical quantities were performed with the help of digital panning and color mapping.
\end{abstract}

\begin{abstract}
Along with technical applications, shock waves are widely used in fundamental scientific research as they are currently a basic experimental technique used to study thermodynamic properties of the matter in the area of high-, and super-high pressures, and also rheological behavior in conditions of high-rate strain deformation. Significantly greater amplitudes of shock waves can be generated with the explosive systems that use the principle of detonation wave convergence. In spherical geometry, pressure at the detonation wave front is continuously growing as far as move to the center. Therefore, experiments on the explosive spherical compaction are a good tool to study materials under super-high pressures [1].

This paper presents the results of metallographic examination of a thick-wall spherical shell made from uranium alloyed with iron and germanium. The original shell geometry and loading mode were similar to those in the research involving uranium alloyed with molybdenum reported at the $\mathrm{X}^{\text {th }}$ International Ural Workshop (Russia, February 25 - March 3, 2013) [2].

A metallographic meridional micro-section of the test sphere was investigated using light microscopy and raster electron microscopy, as well as X-Ray diffraction study and hardness measurement in comparison with the initial state of the material. For analysis, experimental results were visualized using the color mapping method in the same way as it was proposed for studying spherical samples made from uranium alloys [2 - 4].

First, the sample was examined prior to its explosive loading. The material had a threephase structure (Fig. 1). The X-ray energy dispersive microanalysis and X-Ray diffraction study was used to confirm chemical composition of each phase and also inclusions (Fig. 2).
\end{abstract}

*Corresponding author: bad1331@vniitf.ru 

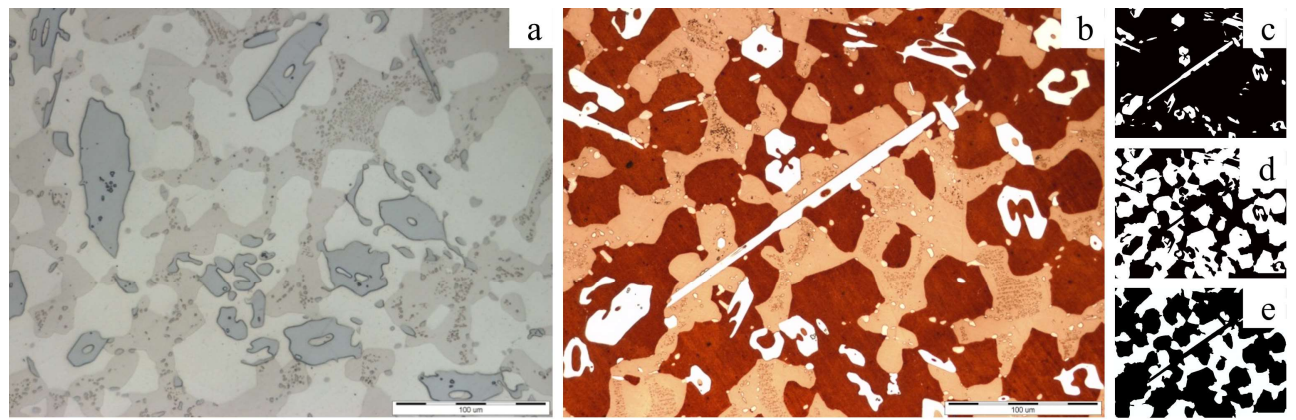

Fig. 1. Structure of the sample in the initial condition after: a - electrochemical polishing and etching; $b$ - cathode plasma etching with argon ions; $c$-e — binary masks of phases 1-3 respectively used to determine their volumetric fraction.
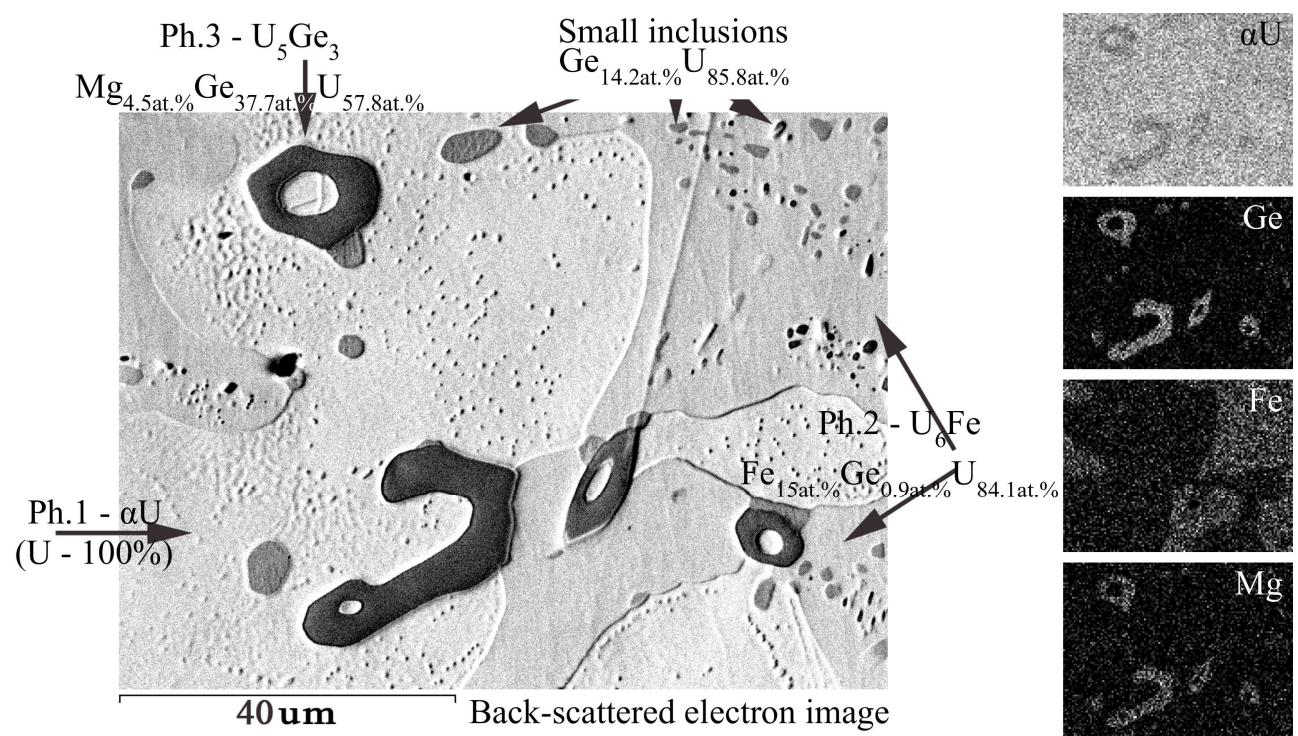

Fig. 2. Chemical elements distribution maps given by the x-ray energy dispersive spectrometer.

The maps on fig. 2 show that phase 1, light-colored and easy etchable, consists of uranium in the $\alpha$-phase, phase 2 contains $\mathrm{U}_{6} \mathrm{Fe}$, dark-colored and exhibiting low etchability if compared with phase 1 , and phase 3 contains intermetallic inclusions $\mathrm{U}_{7} \mathrm{Ge}$ or $\mathrm{U}_{5} \mathrm{Ge}_{3}$ having a specific easy-recognizable shape [5]. The phase identification is in good agreement with the results of the performed X-ray phase analysis.

Statistically valid measurements on bright-field images showed that volumetric fraction of the phase 1 was $\sim 52 \%$, phase 2 was $\sim 37 \%$, and phase 3 was $\sim 11 \%$. Distribution of uranium-germanium inclusions by length is satisfactorily described by the lognormal law with parameters $\mu=12 \mu \mathrm{m}$ and $\sigma=2.4 \mu \mathrm{m}$. Microhardness was measured for each phase. The measurements show that inclusions of phase 3 is the most solid phase with the widest statistical straggling $\left(524 \pm 78 \mathrm{~kg} / \mathrm{mm}^{2}\right)$. The least solid phase is phase $1\left(321 \pm 58 \mathrm{~kg} / \mathrm{mm}^{2}\right)$. The phase 3 exhibits intermediary microhardness $\left(476 \pm 55 \mathrm{~kg} / \mathrm{mm}^{2}\right)$. The material hardness was $335 \pm 24 \mathrm{kG} / \mathrm{mm}^{2}$ with $\sim 140$ measurements in total. However, in view of relatively large area of imprints, these values should be considered as averaged over several structural elements and therefore, they convey an average material hardness. 
A large digital panorama given in fig. 3 was used for microscope examination of the fractured state of meridional cross-section of the shell.

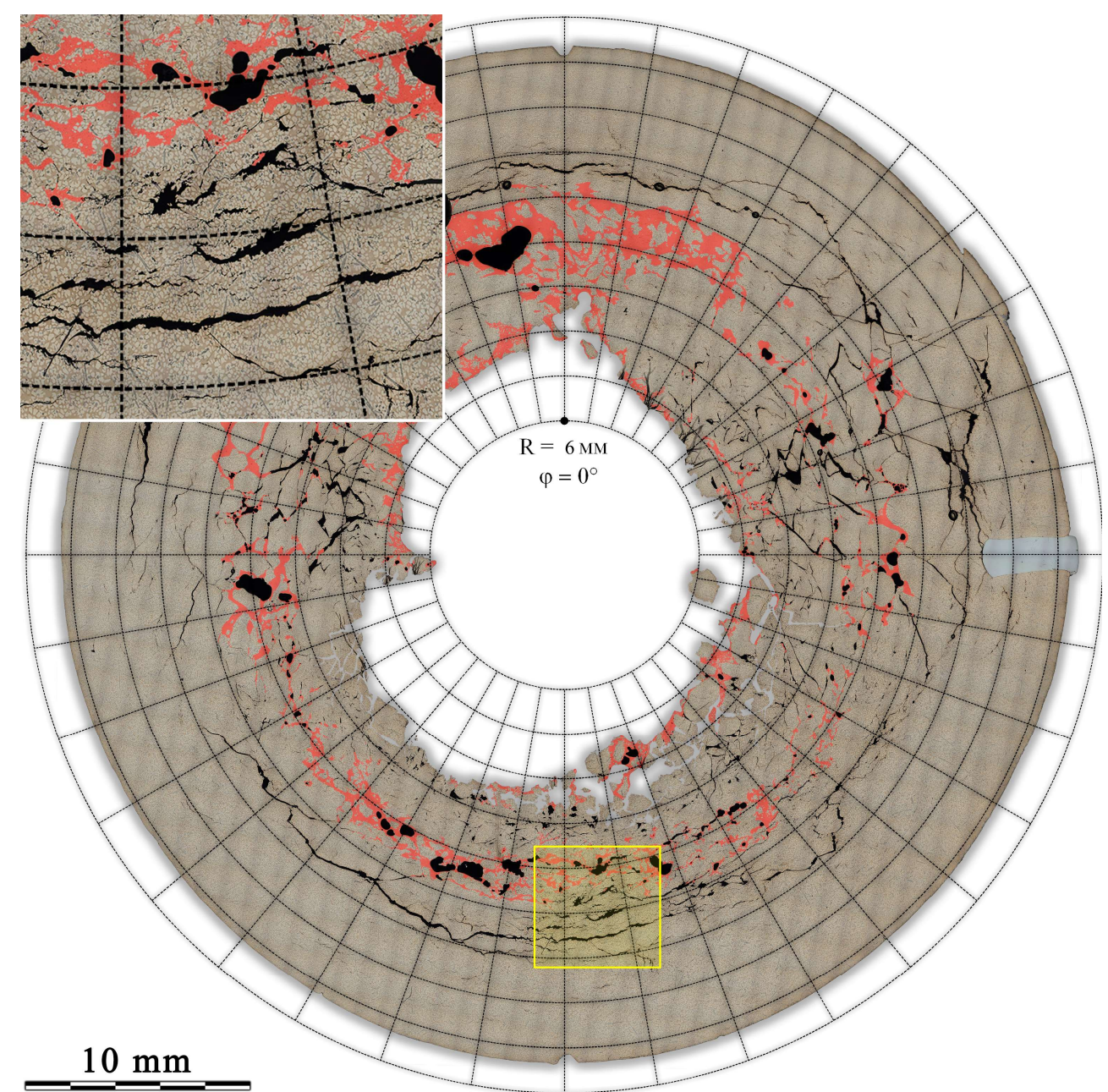

Fig. 3. General view of fractures revealed on the polished surface of the meridional crosssection of the shell. Northern pole is on top. PiP: fractures localization at radii $\mathrm{R} \approx 13 \ldots 16 \mathrm{~mm}$ and $\mathrm{R} \approx 16 \ldots 19 \mathrm{~mm}$.

The fractured area revealed on the meridional cross-section has a symmetrical, approximately concentric structure. Large cracks are evidently located within two rings at radii $\mathrm{R} \approx 13 \ldots 16 \mathrm{~mm}$ and $\mathrm{R} \approx 16 \ldots 19 \mathrm{~mm}$.

The fractured area located most closely to the central cavity is represented by roundedged cracks $(\mathrm{R} \approx 13 \ldots 16 \mathrm{~mm})$ having a shape close to the equiaxial one. Large fractures at these radii are almost absent in the northern segment giving place to single large cavities.

The second group of fractures located at radii $\mathrm{R} \approx 16 \ldots 19 \mathrm{~mm}$ is represented by extended and narrow cracks having up to 30 -mm length and oriented normally to the radius.

Between large cracks of the first and the second groups of fractures one can clearly see multiple smaller cracks having almost the same orientation and exterior.

The cracks reaching the central cavity are essentially oriented at $\sim 45^{\circ}$ to the radius and are observed in any direction from the center. Some of them are widely opened and have a 
more rounded contour than the less opened cracks. The ring with these cracks is limited from the top by radius $\mathrm{R} \approx 12 \mathrm{~mm}$.

Studying the structural condition of the recovered-shell material showed that the structure over almost the entire surface of the metallographic cross-section was similar to that of a nonloaded sample except for the area with fractures localized at radiuses $\mathrm{R} \approx 13 \ldots 16 \mathrm{~mm}$. Here, microstructure of the material is represented by a melted and abruptly crystallized metal having small inclusions in its matrix (Fig. 4a). Appearance, shape, and orientation of individual elements being of another structure type vaguely resemble fractures available within this radii range. Our sample preparation technique and our observation modes revealed no structural elements of this phase (grain boundaries, etc.).
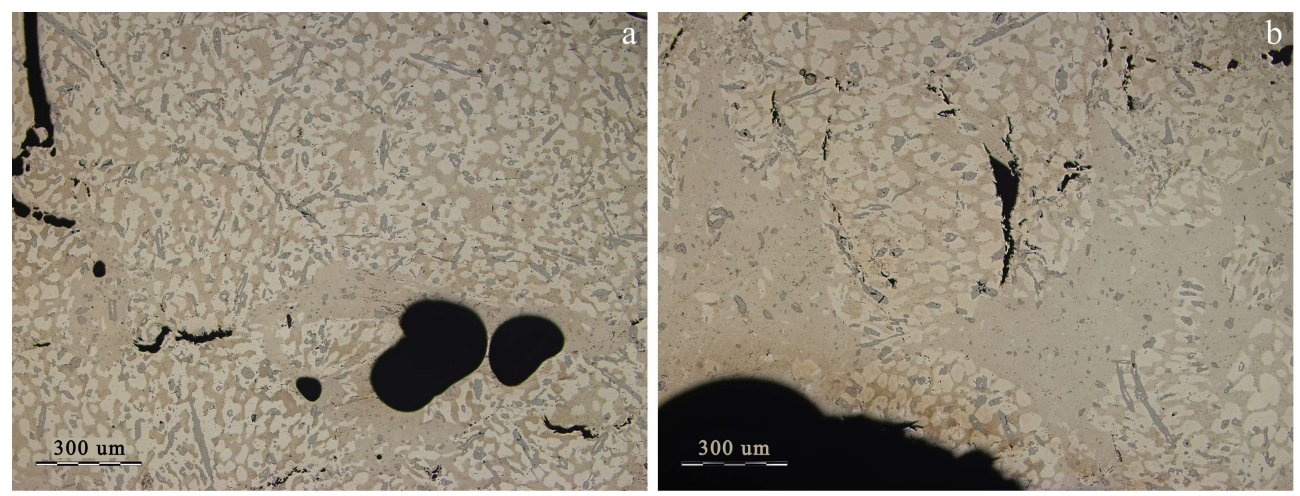

Fig. 4. Structure of the deformed material in the nearest-to-central cavity annular area with fractures localization (a) and material state in the thin layer close to the edge of the central cavity (b).

In Fig. 3, the observed structural difference is shown in red. One can see that this area is at the same radii just as the nearest-to-central cavity area with the annular localization of fractures. In the northern segment with practically no fractures at the given radii, the zone with the structure covers a larger area. Fractures and areas with the changed structure form in total a common annular segment.

This type of the structure was also observed in a thin layer adjacent to the edges of the central cavity along the perimeter. Fig. $4 \mathrm{~b}$ shows that these formations are also arranged as an uncompleted annular segment. Since the structure looks the same both at the edge of the central cavity, and at radii $\mathrm{R} \approx 13 \ldots 16 \mathrm{~mm}$, there is no doubt that structural changes in the given areas followed the same mechanism.

In order to obtain microhardness and hardness distribution, we made and measured 3327 microhardness imprints and 1125 hardness imprints being almost uniformly distributed over the metallographic cross-section. Measurement results in the form of color maps are given in Fig. 5.

Comparison of the hardness and microhardness distribution maps shows that they are similar within a resolution range (not in color space). The microhardness map exhibits more non-uniformity if compared with the hardness map. Common feature in both maps is that microhardness is somewhat higher in the northern segment and somewhat lower in the southern segment at $\mathrm{R} \approx 12 \ldots 18 \mathrm{~mm}$. 


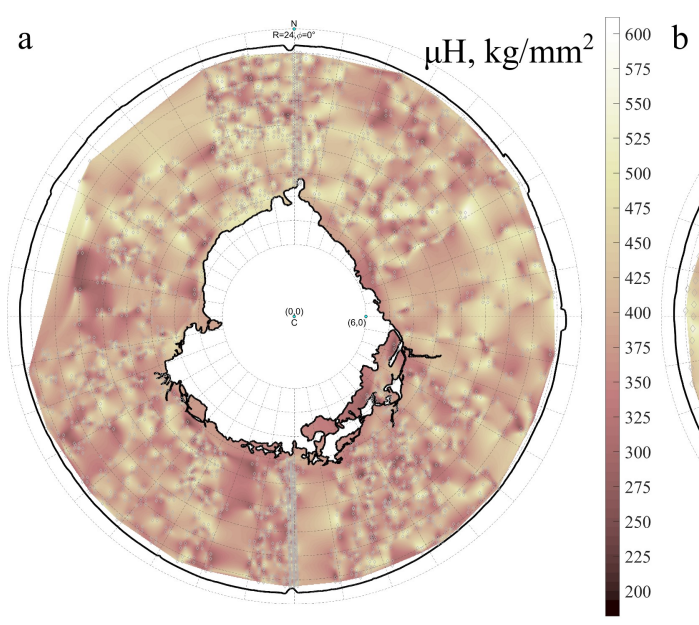

Fig. 5. Color maps of microhardness (a) and hardness (b) distribution over the meridional cross-section of the shell.

Summarizing the experimental results, we can make certain conclusions on the reasons of the observed structural state of the shell.

The pattern of structural changes and fracturing in our test shell is similar to that given by studies of uranium-containing shells at RFNC-VNIITF in recent years.

Correct interpretation of physical changes observed in the material requires understanding of what happens in the shell body under short-term high-intensity loading. The most simple and essential description of events sequence under explosive loading is given in [6].

The first spall occurs in the shell when the converging shock wave is first time reflected from its inner boundary. Then, the spall layer starts moving toward the center almost by inertia thus spending its kinetic energy for plastic deformation and its own heating-up. Attained high temperatures and pressures can result in allotropic and phase transformations (melting, evaporation) of the shell material.

In shells made from fragile materials, the spall layer can fracture into separate fragments during convergence but according to the shear mechanism. Explosion products fail to affect the separated layer, since it is shielded by the rest unfractured part of the shell. If thickness of the spherical explosive layer used for the shell compaction is small, explosion products are quickly released due to free scattering.

Yet, impact of explosion products on the rest part of the shell might be insufficient and the shell will stop during convergence at a certain radius and thus won't "catch up" with the separated layer. If, in order to hinder scattering of explosion products, a heavy housing is placed above the explosive layer with a certain gap, this will ensure that the main part of the shell would "catch up" with the spall layer and this will also help to observe special features in the compaction behavior of the material in the shell that was fractured at a high radius during its convergence to a smaller radius. If the system still has enough energy, one more or even several spalls can be formed in the rest part of the shell according to the above mechanism. However, the follow-on several spalls have no strict localization relative to the radius due to blurring of stress wave fronts, anisotropy, and defects in the material lead [2].

At last stages of shock wave loading, the shell slowly gets compacted up to the full stop. Until the shell is completely cooled down, there is enough time for such low-rate processes as diffusion, recrystallization, and defect annealing to take place.

As for the test shell, one can see that the localized fractures revealed within $\mathrm{R} \approx 13 \ldots 16 \mathrm{~mm}$ and $\mathrm{R} \approx 16 \ldots 19 \mathrm{~mm}$ are nothing but the first and the second spalls, respectively. Note that the first spall was sufficiently recompacted in the northern segment. And 
evidence of cracks recompaction is a structure with traces of metal melting we observed in the previous location of cracks induced by spallation. The southern segment also has evidences of the first spall recompaction. However, the recompaction extent here is somewhat lower due to minor symmetry violations caused by explosive loading.

The fractures that form the second spall are narrow, long, and have distinct rugged edges. Appearance and shape of fractures indicate that the material was fractured mainly according to the fragile mechanism (at least during the second spall formation) and the material itself demonstrated a sufficiently high resistance to fracturing. High strength of the material is most likely conditioned by the fact that the structure is observed to have narrow inclusions of $\mathrm{U}_{5} \mathrm{Ge}_{3}$ making one tenth of the material volume. Our measurements demonstrated that these inclusions are of high hardness and, therefore, high strength. So, they reinforce the loaded material enhancing its capability to resist to the loading applied.

In annular zones of southern and northern segments, local decrease in hardness is observed at the second-spall radius and this is clearly seen in the distribution maps (fig. 5). The decrease is caused by the fracturing-induced relaxation of the stressed material.

Numerous fractures reaching the inner cavity of the shell are located at $\sim 45^{\circ}$ to the radius and this speaks for the shear mechanism that governs formation of fragments in the first spalllayer under convergence.

At the microstructure level, no changes in the material are revealed except for the areas (recompacted cracks in the first spall and areas near the central cavity boundary) bearing traces of a short-term melting of the material.

\section{References}

1. Methods to study materials properties under high-intensity dynamic loading. Monograph under the general editorship of Dr. Sci. in Physics and Mathematics M.V. Zhernokletov, Sa$\operatorname{rov}(2003)$

2. A.S. Aleksandrov, D.A. Belyaev, Yu.N. Zuev, E.A. Kozlov, I.L. Svyatov, E.A. Shestakova, Metallographic study of meridional section of containment made of $U-1.5 \% \mathrm{Mo}-1 \% \mathrm{Zr}$ alloy after explosive loading, abstracts for X International Ural Seminar "Radiation fracture physics of metals and alloys", February 25 - March 3 2013, Kyshtym, Russia, pp. 38-39 (2013)

3. E.A. Kozlov, D.A. Belyaev, Yu.N. Zuev, I.L. Svyatov. Spall and shear deformation of U1,5\% Mo shell at explosive loading, Deformation and destruction of materials, 2, pp. 26-30 (2012)

4. Yu.N. Zuev, D.A. Belyaev, E.A. Kozlov, I.V. Podgornova, I.L. Svyatov. Metallographic study of uranium shells after shock-wave loading using color mapping method, abstracts for IX International Ural Seminar "Radiation fracture physics of metals and alloys", February $20-26$ 2011, Kyshtym, Russia, p. 53 (2011)

5. State diagrams of binary metallic systems. Reference book under the general editorship of N.P. Lyakishev, academician of RAS. Volumes 1-4. Moscow (1996)

6. E.A. Kozlov et alt. Spall and shear rupture in spherically converging shells of iron and steels. Measurement of energy and residual deformations. Measurement of energy and residual deformations, Deformation and rupture of materials, 11 (2008)

7. V.S. Nikiforovsky, E.I. Shemyakin. Dynamic rupture of solid bodies. Novosibirsk, "Nauka" (1979) 